\title{
Investigation of the Iron(II) Release Mechanism of Human H-Ferritin as a Function of $\mathrm{pH}$
}

\author{
Davide Sala, $^{\dagger}$ Silvia Ciambellotti, $^{\dagger}$ Andrea Giachetti, ${ }^{\ddagger}$ Paola Turano, ${ }^{\dagger, \S_{\odot}}$ and Antonio Rosato ${ }^{*},, \S_{\odot}$ \\ ${ }^{\dagger}$ Magnetic Resonance Center (CERM), University of Florence, Via Luigi Sacconi 6, 50019 Sesto Fiorentino, Italy \\ ${ }^{\ddagger}$ Consorzio Interuniversitario di Risonanze Magnetiche di Metallo Proteine, Via Luigi Sacconi 6, 50019 Sesto Fiorentino, Italy \\ ${ }^{\S}$ Department of Chemistry, University of Florence, Via della Lastruccia 3, 50019 Sesto Fiorentino, Italy
}

\section{Supporting Information}

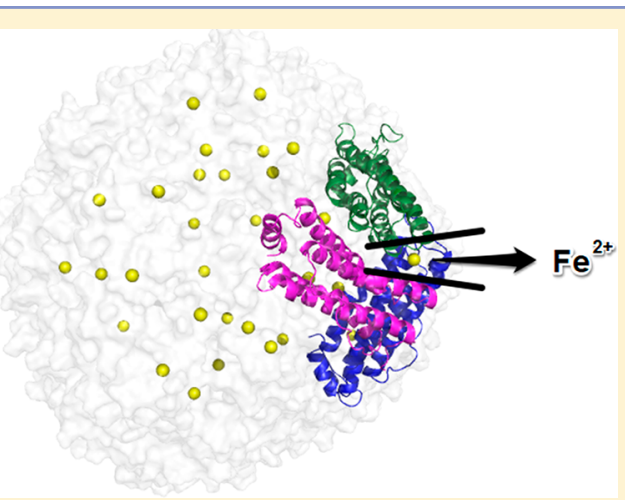

ABSTRACT: We investigated the kinetics of the release of iron(II) ions from the internal cavity of human $\mathrm{H}$ ferritin as a function of $\mathrm{pH}$. Extensive molecular dynamics simulations of the entire 24-mer ferritin provided atomiclevel information on the release mechanism. Double protonation of His residues at $\mathrm{pH} 4$ facilitates the removal of the iron ligands within the C3 channel through the formation of salt bridges, resulting in a significantly lower release energy barrier than $\mathrm{pH} 9$.

\section{INTRODUCTION}

Maxi ferritins are 24-mer nanocage structures that self-assemble from 4-helix bundle subunits. ${ }^{1}$ The resulting structure has octahedral symmetry. In correspondence to the C3 and C4 symmetry axes there are two different channels connecting the bulk solution to the inner cage cavity (Figure 1). The two types of channels differ in their size and chemical properties. ${ }^{2-5}$ In vertebrate ferritins, the eight $\mathrm{C} 3$ channels constitute the entry points of iron(II) ions, ${ }^{2,6}$ which transit under the form of hexahydrated ions under the effect of the electrostatic gradient generated by carboxylate groups at the inner end of the channels. The iron release mechanism in biomineralized ferritin is less characterized. ${ }^{7}$ In vitro, release experiments generally involve the addition of a reducing agent in the presence of chelators. $^{8-11}$ The process in vivo might be associated with cage disassembly, ${ }^{12,13}$ or it could depend on specific acidic environments. ${ }^{14,15} \mathrm{H}$-ferritin enters cells via receptor-mediated endocytosis mediated by Transferrin Receptor 1 (TfR 1$){ }^{16}$ Binding of $\mathrm{H}$-ferritin to TfR 1 results in the uptake of $\mathrm{H}$-ferritin into endosomes and lysosomes in a dynamic process. During their maturation from early to late endosomes, the $\mathrm{pH}$ inside these organelles is in the 6.0-4.8 range whereas in lysosomes the $\mathrm{pH}$ can drop to values around $4.5 .{ }^{17,18}$

Our kinetic measurements on human $\mathrm{H}$-ferritin in the presence of different reductants showed that iron release has different kinetic profiles but with the same distinct $\mathrm{pH}$ dependence (Figure 2A and $\mathrm{B}$ ). In particular, lowering the $\mathrm{pH}$ from 9.0 to 4.0 increased significantly the amount of iron discharged. We used MD simulations to identify the parts of the protein responsible for the interaction with the iron ions during the release process. We focused on the processes occurring at $\mathrm{pH} \mathrm{4,} \mathrm{where} \mathrm{iron} \mathrm{release} \mathrm{is} \mathrm{enhanced,} \mathrm{and}$ compared them to $\mathrm{pH}$ 9. Furthermore, the umbrella sampling method was applied to estimate the corresponding free-energy barriers. At $\mathrm{pH} \mathrm{4}$, all His residues are doubly protonated, whereas all carboxylates are deprotonated. Consequently, the internal cavity of ferritin provides an environment whose electrostatic potential is close to neutrality due to the balance of the charges of the doubly protonated His side chains (positive) and of the carboxylate side chains (negative). Therefore, the electrostatic surface of the protein's cage does not stabilize significantly metal ions in the absence of mineralization. The iron(II) ions thus respond readily to the gradients of electrostatic potential leading them toward other regions of the structure where negatively charged residues are spatially clustered. Such gradients drive the iron ions toward two main locations: the ferroxidase catalytic sites and the $\mathrm{C} 3$ channels (movie S1). The same behavior takes place at $\mathrm{pH} 9$ despite the fact that in each chain nine out of ten His residues are neutral and thus the electrostatic charge within the cavity at this $\mathrm{pH}$ is negative. In this work, we obtained evidence that the $\mathrm{C} 3$ channels are the pathway for the release of iron(II) ions from the cavity to the bulk solution ${ }^{19}$ (movie S2). Our MD simulations provide a detailed atomic-level view of the mechanism of iron release from ferritin, which was still missing. ${ }^{20}$

\section{RESULTS}

The eight C3 channels are formed by three symmetry-related motifs, ${ }^{21}$ specifically helix $\alpha 4$-loop- $\alpha 3$, from as many chains (A, $B$, and $C$ ). Within each individual channel, two nearby rings formed by symmetry-related Asp and Glu side chains define the binding site to which the iron ions move from the internal cavity (Figure 1). The side chains of the Asp131 residues define

Published: August 30, 2017 

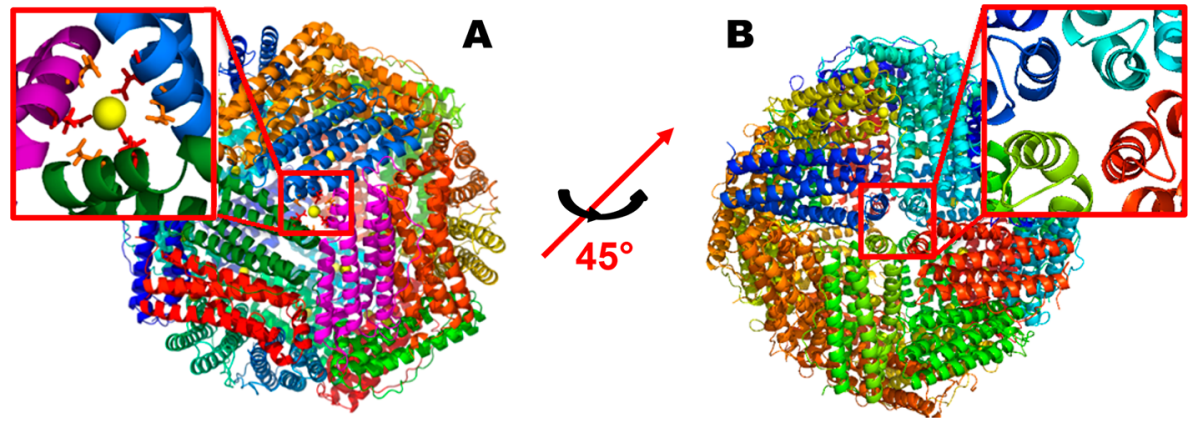

Figure 1. Structure of human $\mathrm{H}$-ferritin viewed down the $\mathrm{C} 3$ and $\mathrm{C} 4$ axes, related by a $45^{\circ}$ rotation around the red axis. Each subunit is colored differently. Iron ions inside the $\mathrm{C} 3$ channel are shown as yellow spheres. (A) The C3 channel inside view is illustrated in the red box on the left. Glu134 and Asp131 side chains are shown as red and orange sticks, respectively. (B) The C4 channel is illustrated in the red box on the right.

A

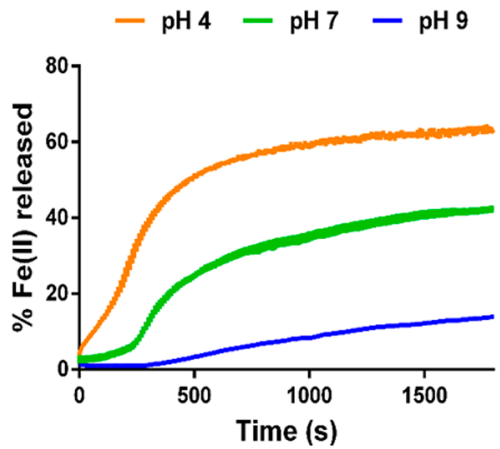

B
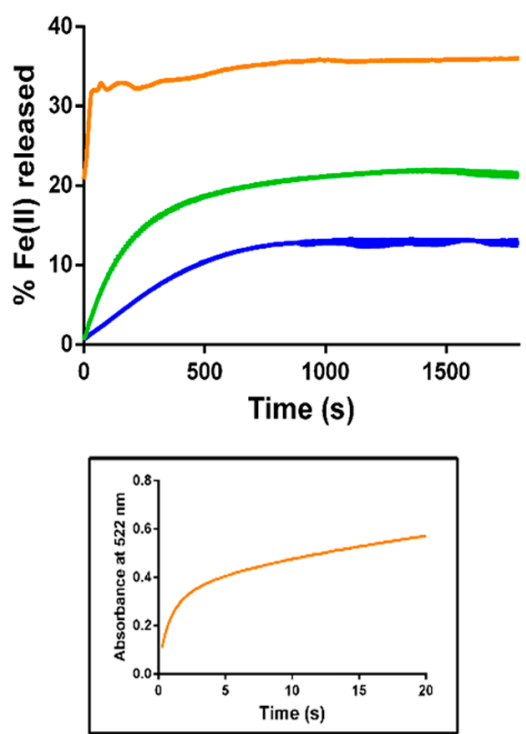

Figure 2. Iron(II) release in human $\mathrm{H}$-ferritin at different $\mathrm{pH}$ conditions. The plots show the percentage of iron(II) released over $1800 \mathrm{~s}$ at $\mathrm{pH} 4,7$, and 9 by human holo $\left(480 \mathrm{Fe}^{2+}\right.$ per cage $) \mathrm{H}$-ferritin. The curves represent the mean \pm SEM of six independent analyses. The student's $t$ test indicated that the curves are significantly different $(P<0.05)$. The graph shows the iron(II) release in the presence of (A) NADH/FMN reductants or (B) sodium dithionite. (B inset) First seconds of the kinetics at $\mathrm{pH} 4$ in the presence of dithionite (represented as absorbance at $522 \mathrm{~nm}$ over time) acquired with a stopped-flow spectrophotometer, which allowed us to measure the initial points of the reaction that are lost with manual mixing.

a first ring that is closer to the internal cavity of ferritin, whereas the side chains of the Glu134 residues define a second ring, further from the internal cavity, and thus closer to the protein surface. In our simulation at $\mathrm{pH} 4$, three of the eight $\mathrm{C} 3$ sites become populated at different time points by a single iron(II) ion. At $\mathrm{pH} 9$ four sites become populated. Typically, the iron ion reached the site within the first nanoseconds of the simulation. The fact that not all eight channels become populated can be due to the relatively low number of ions introduced in the cavity ( 31 ions) as well as to the initial position of each ion, e.g., because of the different relative distance between each ion and the closest ferroxidase vs C3 sites. In addition, the local conformations of the eight sites in the initial structural model, built by aligning the human $\mathrm{H}$-chain on the bullfrog crystal structure ${ }^{22}$ (PDB entry 4DAS), are not identical.

Before the iron ion actually reaches the C3 site, the Asp131 side chains move apart thereby allowing the metal ion to get into the space between the two rings of carboxylates (Figure S1). Within the site, the iron can be in proximity to the side chains of the Glu134 and Asp131 residues (Figure 1). Once inside, the metal ion moves toward the exit of the channel and, in so doing, it transitions between different coordination configurations. As an example, Figure 3 shows how the coordination of iron in one of the $\mathrm{C} 3$ sites at $\mathrm{pH} 4$ is affected by the dynamics of Glu134(C) and its interactions with neighboring histidinium (i.e., the cationic form of His) side chains. When the iron enters the site, it is at a short distance from all three Glu residues. However, after only a few ns Glu134(C) changes conformation and moves away from the metal (Figure 3). This is triggered by the subsequent formation of two salt bridges involving the negatively charged carboxylate of Glu134(C). The first salt bridge is formed with the positively charged side chain of His128(A), which is later (after about 1 ns) displaced by His136(A). The latter event causes the carboxylate of Glu134(C) to rotate away from the iron ion and toward the cavity of ferritin (Figures 3 and S2). The other two Glu134 residues in the site (chains A and B) are released only when the iron ion exits the C3 channel (Figure 4).

The pairwise distances among the three Glu134 residues of the three chains define the process of opening of the channel. For the same $\mathrm{C} 3$ site described previously, the three distances are similar in the initial configuration of the ferritin structure (Figure 4C). Upon the arrival of the iron ion in the site, they evolve into one shorter (about $6 \AA$ ) and two longer distances (8-8.5 $\AA$ ). The situation changes further upon the rearrangement of Glu134(C) described in the previous paragraph and Figure 3. After this event, the distance between Glu134(A) and Glu134(B) oscillates between 6 and $8 \AA$ but is typically closer to the lower part of this range, whereas the two distances involving Glu134(C) rapidly increase above 8 Å reaching peaks 


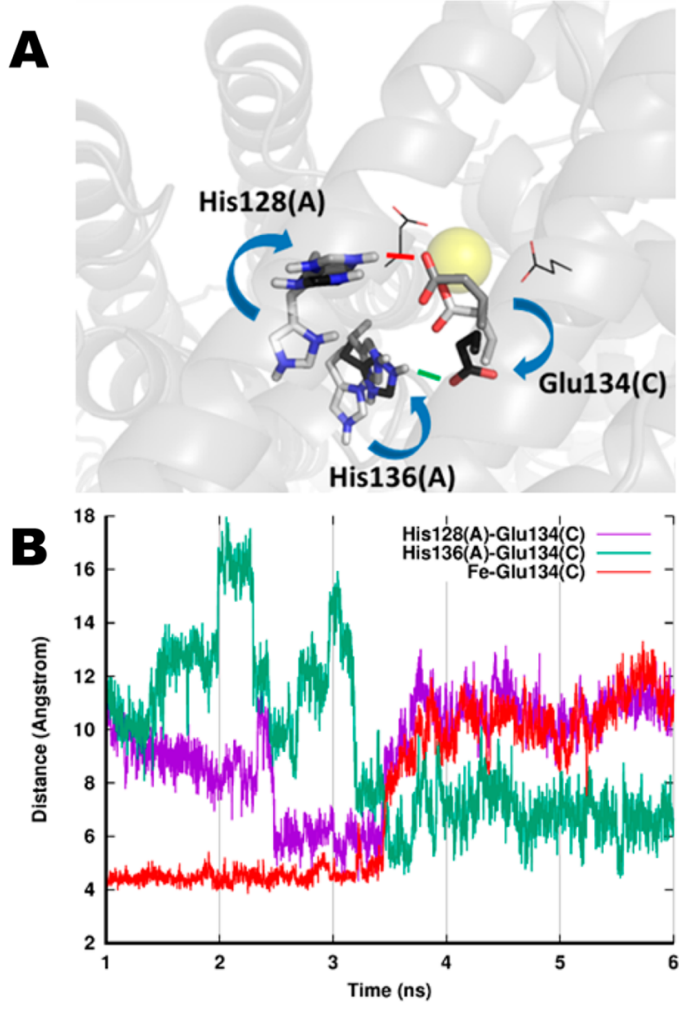

Figure 3. Switch of iron coordination in a C3 site. (A) Overlap of three snapshots at $2.36 \mathrm{~ns}$ (white), $3.38 \mathrm{~ns}$ (gray), and $3.63 \mathrm{~ns}$ (black). Residue side chains involved in the process are shown as sticks. The red dash indicates the salt bridge between His128(A) and Glu134(C) at $3.38 \mathrm{~ns}$ that weakens the iron(II)-Glu134(C) interaction. The green dash shows the salt bridge between His136(A) and Glu134(C) that is formed at $3.63 \mathrm{~ns}$, just after iron(II)-Glu134(C) detachment. (B) Iron(II)-Glu134(C), His128(A)-Glu134(C), and His136(A)Glu134(C) distances for the first channel. His-Glu distances refer to the $\mathrm{H} \varepsilon$ and $\mathrm{C} \delta$ atoms of the His and Glu residues, respectively.

of $12 \AA$. While the iron ion is in the site, the carboxylate $\mathrm{O} \varepsilon$ oxygen atoms do not form consistent hydrogen bonds to donor groups in spatial proximity and thus remain close to each other due to electrostatic interaction with the metal ion. At $\mathrm{pH} 4$ this structural configuration evolves during the MD simulation, eventually leading to the release of the iron ion (see below). Instead, the corresponding configuration at $\mathrm{pH} 9$ remains stable throughout the entire simulation (e.g., Figures S3 and S4).

The protein dynamics at $\mathrm{pH} 4$ leads to conformations featuring relatively long-lived salt bridges involving the Glu134 and the doubly protonated His 118 residues in the channel. This is apparent between 5 and $20 \mathrm{~ns}$ of the simulation (Figure 4A and $\mathrm{B}$ ). The presence of these salt bridges can be conveniently monitored by looking the hydrogen bond persistence between the side chains (Figure 4D).

The iron ion is released when the $\mathrm{A}$ and $\mathrm{B}$ chains simultaneously feature long-lived hydrogen bonds, between 15 and $20 \mathrm{~ns}$ (Figure 4B and D). This configuration reinforces the correlation between the dynamics of the protein structural environment and the dynamics of the Glu134 side chains. In addition, there is a partial compensation of the negative electrostatic charge of the carboxylates of the Glu134 residues. Eventually, the iron ion escapes the $\mathrm{C} 3$ binding site and irreversibly (in our simulations) diffuses into the bulk solution (Figure 4). The channel closes after the iron release and Glu134(C) comes back to the starting orientation (Figure 4C and movie S3). When the His residues are singly protonated, the aforementioned electrostatic mechanisms are no longer possible. Indeed, at $\mathrm{pH} 9$, where all His are singly protonated, the iron ions move quickly toward the nearest $\mathrm{C} 3$ channel from their starting position, but the Asp and Glu rings do not open and the ions remain blocked in the site (Figures S3 and S4). Clearly, the double protonation of the His residues is crucial to enable an effective mechanism for the release of the iron ions. Notably, at pH 7 the majority of the His side chains are doubly protonated thereby suggesting that the mechanism described at $\mathrm{pH} 4$ could be relevant also at neutral $\mathrm{pH}$. In the final part of the simulation at $\mathrm{pH} \mathrm{4}$, although the iron ion has already left the protein His128(A) closes the gap with Glu134(C) as previously observed (Figure 3). Thus, the Glu134 distances increase and the channel opens again (Figure 4C and movie S3 at about $100 \mathrm{~ns}$ ) despite the absence of the metal ion.

As mentioned, during our simulation at $\mathrm{pH} 4$ we observed two iron ions being released through two different channels in the bulk solution. In the second channel, the entry of the iron occurs after a few ns and all three Glu134 side chains are recruited to form the coordination sphere, which remains stable for nearly $40 \mathrm{~ns}$ of trajectory (Figure S5). The distance of the three Glu134 side chains from one another are not all identical, with two pairs at about $6 \AA$ and the third (Glu134(A) and Glu134(B)) at $7 \AA$ for nearly 20 ns. Afterward, there is a rearrangement of the site, with the distance between Glu134(B) and Glu134(C) remaining at $6 \AA$, whereas the other two distances increase up to $8 \AA$ until 40 ns. Shortly after $40 \mathrm{~ns}$ of simulation, Glu134(B) rearranges similarly to what we described for Glu134(C) in the first channel with the exception that His136(A) is closer to Glu134(B) than His128(A) (Figure S6). Nevertheless, the simultaneous proximity of both histidinium side chains allows Glu134(B) to move away from the metal ion. After this event, the distance between the side chain of Glu134(A) and the iron ion oscillates rapidly in the range 4.5-8.0 $\AA$. Instead, the iron-Glu134(C) distance remains within 4.5-5.0 $\AA$, with sporadic transitions at longer distances when the iron-Glu134(A) falls at $5.0 \AA$ or less (e.g., see around $80 \mathrm{~ns}$ in Figure S5). In summary, within this second channel the iron ion is mostly bound to Glu134(C). Occasionally the iron ion moves from Glu134(C) toward Glu134(A), then moves back. On top of this, Glu134(A) itself is moving back and forth with respect to the other two glutamates. These extensive structural fluctuations correspond to a situation where the $\mathrm{C} \delta$ atoms of the three Glu residues of the $\mathrm{C} 3$ site have changed their configuration from a roughly triangular distribution in space to a distribution closer to linearity. The escape from this situation is mediated again by the formation of a persistent salt bridge to positively charged His118 residues (Figure S7). This reinforces the link between the motions of Glu134(C) and Glu134(A), and the rest of the protein chain while simultaneously reducing their electrostatic interaction with the iron ion. In this way, the ion can displace more from its position and eventually escapes from the channel.

The umbrella sampling method ${ }^{23}$ was applied to measure the free-energy of the iron(II) release process through the C3 channel. In addition to simulations at $\mathrm{pH} 4$ and $\mathrm{pH} 9$, we prepared a chimeric variant of ferritin in which the protonation state at $\mathrm{pH} 9$ was altered by doubly protonating the side chains of the three histidines that play a crucial role in the release process at $\mathrm{pH} 4$ : His118, His128, and His136. At the starting point of the umbrella simulations, the metal ion is located inside the ferritin cage. Then, in all the simulations, the 

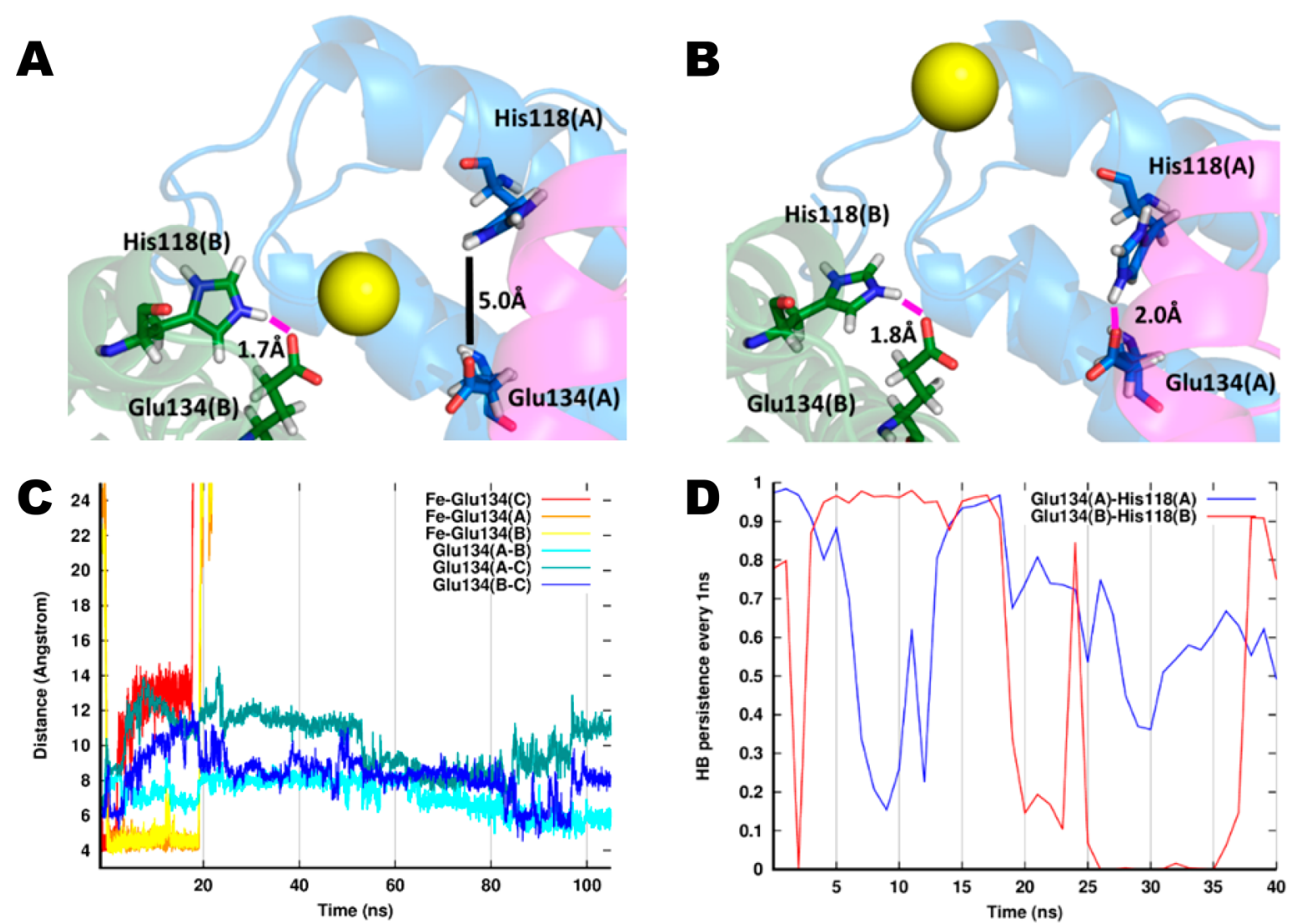

Figure 4. Iron release after the formation of two Glu134-His118 salt bridges in the first channel. Chain A is in blue; chain B is in green. The metal ion is shown as a yellow sphere. (A) Glu134(A) is too far from His118(A) to make a salt bridge, and the iron(II) ion is still coordinated. (B) His118(A) moves in proximity of Glu134(A), and a salt bridge is formed. The electrostatic attraction between the carboxylate $\mathrm{O} \varepsilon$ oxygen atoms and the metal is interrupted and the iron(II) ion is free to leave the channel. (C) Iron-Glu134 and Glu134-Glu134 distances for one of the three C3 channels populated by iron(II). For Glu residues, all distances refer to the C $\delta$ atoms. Iron(II)-Glu distances are shown using hot colors, Glu-Glu distances are shown using cold colors. The release of iron occurs at about 19 ns (D) The plot shows the persistence of Glu134-His118 hydrogen bonds on a window of $1 \mathrm{~ns}$, normalized by the number of frames in the window. The persistence increases for both hydrogen bonds between 15 and $19 \mathrm{~ns}$ of the simulation, i.e. immediately before iron release.

electrostatic gradient pushes rapidly the iron ions inside the $\mathrm{C} 3$ channel. Once the metal is inside, the free-energy profile at $\mathrm{pH}$ 4 reaches the minimum at $6.2 \AA$ from the innermost part of the channel, shifted by about 4 and $4.5 \AA$ toward the exit with respect to the minima observed for wild-type and chimeric ferritin at $\mathrm{pH} 9 \mathrm{WT}$, respectively (Figure 5). In fact, the rings formed by the Asp131 and Glu134 residues (Figures 4C and $\mathrm{S} 1$ ) of the three chains can open dynamically at $\mathrm{pH} 4$, allowing the metal ion to move deeper in the channel (Figure 5A). On the contrary, the iron(II) ion at $\mathrm{pH} 9$ is coordinated closer to the cavity, where negatively charged residues are spatially clustered (Figure 5B). The free-energy minimum for the chimeric ferritin at $\mathrm{pH} 9$ is in a position similar to the WT protein, despite the metal coordination is less compact and some electrostatic interactions with the carboxylates are disrupted by the protonated His residues (Figure $5 \mathrm{C}$ ). We can conclude that the double protonation of the side chain rings of only the three His residues (His118, His128, and His136) is not sufficient to restore the channel opening observed at acidic $\mathrm{pH}$. Notably, at $\mathrm{pH} 4$ the energy needed to discharge the iron(II) ion or to attract it in the channel are very similar, not exceeding $2 \mathrm{kcal} / \mathrm{mol}$. Instead, for the WT at $\mathrm{pH} 9$ the energetic barrier for the escape from the binding site within the C3 channel is somewhat higher, reaching a plateau of 15.3 $\mathrm{kcal} / \mathrm{mol}$ around $13.5 \AA$ (Figure S8). Introducing the double protonation for the three key His residues at $\mathrm{pH} 9$ lowers the energy needed to leave the channel to just about $3 \mathrm{kcal} / \mathrm{mol}$.

\section{DISCUSSION AND CONCLUSIONS}

In summary, at both $\mathrm{pH} 4$ and $\mathrm{pH} 9$ the iron ions are rapidly attracted toward the $\mathrm{C} 3$ channels by the electrostatic gradient due to the Glu and Asp side chains that form the C3 site. The Asp residues move apart to allow the metal ion to enter the site, where three Glu side chains are available to coordinate it. This coordination remains stable throughout the simulation at $\mathrm{pH} 9$, whereas at $\mathrm{pH} 4$ we observed for two distinct channels the release of the iron ion into the bulk solution. Overall, the iron release mechanism is very similar for both channels. This process occurs in two main steps (Figure 6): first, the iron ion coordination switches from three to two glutamate side chains (Figure 6A and B). This switch is triggered by the electrostatic attraction between one of the Glu134 side chains and two positively charged histidine residues (His128 and His136). The new orientation of Glu134 is stabilized by His 136 through a salt bridge. In the second step, the iron ion is released when its electrostatic interaction with the carboxylate $\mathrm{O} \varepsilon$ oxygens of the two coordinating Glu134 side chains is interrupted by an increased interaction between these side chains and nearby residues (Figure 6C and D). In this respect, the lifetime of the intrachain Glu134-His118 hydrogen bonds is a useful indicator. Figure 4 suggests that this increase in lifetime is effective for iron release only when it involves at least both Glu residues concurrently. Indeed, the enhanced lifetime of the hydrogen bonds formed by the Glu134 residue from a single chain observed at 5-15 ns in the first channel does not lead to the release of the iron ion. A similar situation occurs in the second channel in the range 65-80 ns. The iron release 

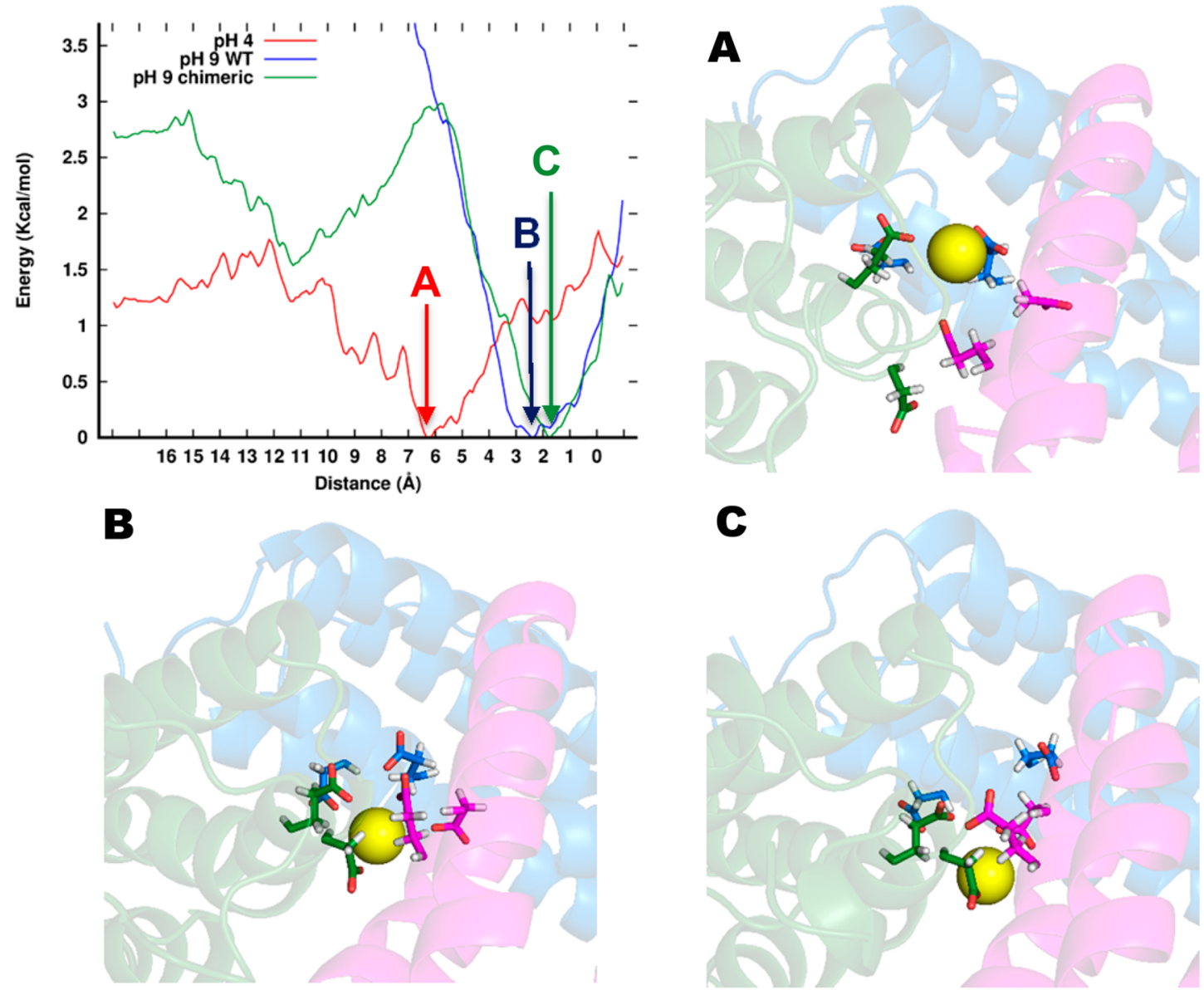

Figure 5. Free-energy profiles along the iron(II) exit coordinate (distances increase from the innermost to the outer part of the channel) through the C3 channel. The free-energy profiles are obtained from the weighted histogram analysis of the umbrella sampling trajectories performed with biased harmonic potential (force constant set at $15 \mathrm{kcal} / \mathrm{mol} \AA^{2}$ ) for $\mathrm{pH} \mathrm{4,} \mathrm{pH} 9 \mathrm{WT}$, and $\mathrm{pH} 9$ chimeric (histidine 118, 128, and 136 are doubly protonated as $\mathrm{pH} 4$ ). The free-energy minima are indicated with arrows, the corresponding metal ion coordinations are shown in pictures $\mathrm{A}, \mathrm{B}$, and C. The iron(II) ion is shown as a yellow sphere, Glu134 and Asp131 side chains are shown as sticks, and each chain is colored differently.

mechanism at $\mathrm{pH} 4$ leverages the favorable electrostatics of the doubly protonated His residues enhancing the effectiveness of the stochastic events that bring Glu134 and His118 in proximity for two protein chains simultaneously.

Based on the umbrella sampling simulations we could estimate the free-energy profiles of the initial configurations. Two main things are noteworthy: first, the position of the minima differ by about $4 \AA$ between $\mathrm{pH} 4$ and $\mathrm{pH}$ 9, denoting a different accessibility of the channel. Second, the energy barrier is somewhat higher at $\mathrm{pH} 9$ than $\mathrm{pH}$ 4. Although all the His residues are doubly protonated at $\mathrm{pH} 4$, three of them are particularly influent in the release process: His118, His128, and His136. We thus leveraged the unique opportunity offered by molecular dynamics simulations to build and study chimeric ferritins to obtain a deeper description of the iron(II) release mechanism. The double protonation of these three His residues introduced in the chimeric form of ferritin at $\mathrm{pH} 9$ could not restore completely the mechanism for the entry of the iron(II) ion in the $\mathrm{C} 3$ channel but achieved a nearly full reduction of the energetic barrier for the escape from the binding site into the bulk solution. In conclusion, the positive charges on the side chains of the His118, His 128, and His 136 residues proved to be crucial to weaken the metal coordination by compensating the negative charges of the Asp131 and Glu134 side chains inside the C3 channel. Instead, only the overall electrostatic contribution of the double protonation of all $\mathrm{His}$ at $\mathrm{pH} 4$ could trigger the dynamic opening of the binding site, thus significantly shifting the position of the coordinated iron(II) ion toward the exit of the channel with respect to $\mathrm{pH}$ 9. In fact, there is a lower energetic barrier to discharge the iron ion in chimeric than in WT ferritin at $\mathrm{pH}$ 9, even though the position of the minimum within the $\mathrm{C} 3$ channels remained the same (Figure 5).

At variance with human $\mathrm{H}$-ferritin, the $\mathrm{C} 3$ channels are not the entry/exit channels for iron ions in bacterioferritins and bacterial ferritins. These bacterial systems exploit the so-called B-pores instead, ${ }^{24,25}$ which are absent in eukaryotic ferritins. For example, in the ferritin of Pseudomonas aeruginosa the threefold pores are lined with side chains of alternating charge. ${ }^{26}$ Indeed, in the $\mathrm{pH} 6.0$ structure of this system, a negatively charged sulfate ion is found within the $\mathrm{C} 3$ channel.

The described $\mathrm{pH}$-dependent mechanism of iron release from ferritin might help defining the role of the acidic environment of endosomes and lysosomes in modulating iron release within these organelles. Encapsulation in endosomes is proposed to be relevant for $\mathrm{H}$-ferritin internalization via the TfR 1 receptor ${ }^{16}$ and therefore plays a role for the use of ferritin as a drug nanocarrier targeting cancer cells overexpressing TfR1. The delivery of ferritin to lysosomes, instead, has been proposed as a key role in controlling iron cellular homeo- 

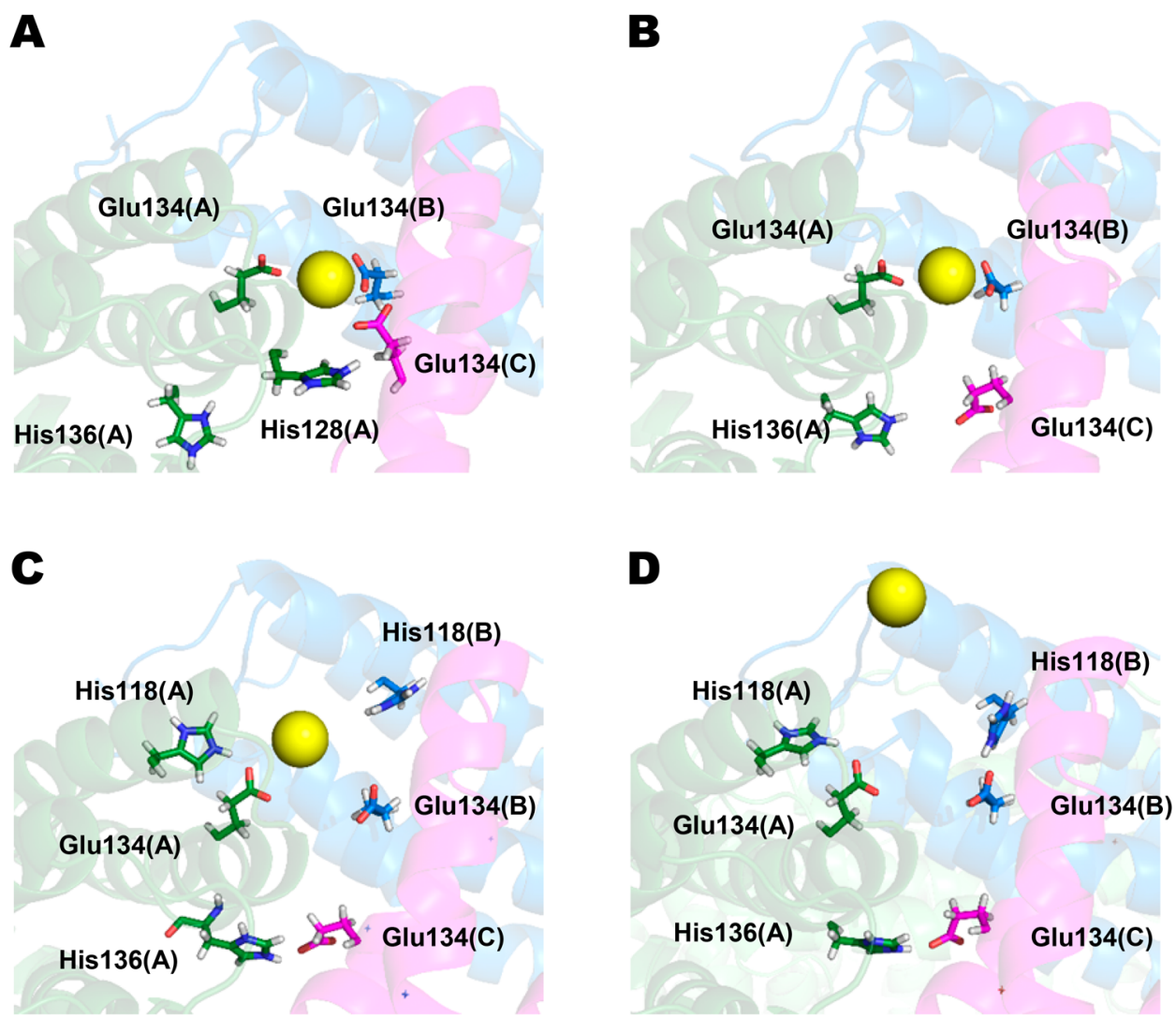

Figure 6. Summary of the crucial steps for iron(II) ion release from C3 channels at $\mathrm{pH} 4$. Each chain is colored differently, and the metal ion is shown as a yellow sphere. Residue side chains involved in the process are shown as sticks. (A) Snapshot at 3.00 ns. The ion is coordinated by three Glu134 residues. His128(A) is approaching the Glu134(C) residue to rip it off from the metal. (B) Snapshot at 3.63 ns. The Glu134(C) is now oriented toward the protein cavity stabilized by His136(A); thus, the ion is now coordinated just by two Glu134 residues. (C) Snapshot at 19.05 ns. During the five nanoseconds before the ion release the interactions between Glu134 and His118 increase, disrupting the metal-carboxylate coordination. (D) Snapshot at 19.24 ns. The simultaneous formation of salt bridges between Glu134 and His 118 causes the iron ion release from the channel.

stasis; ${ }^{15}$ the interplay between biomineral solubilization upon iron reduction and ferritin degradation at this level is not yet clear, and the observed cage-assisted release at low $\mathrm{pH}$ might also come into play.

\section{ASSOCIATED CONTENT}

\section{S Supporting Information}

The Supporting Information is available free of charge on the ACS Publications website at DOI: 10.1021/acs.jcim.7b00306.

Experimental and computational methods and additional figures (PDF)

Movie of a C3 channel, along the whole trajectory, from the protein's cage point of view (MPG)

Movie of the iron(II) ion leaving cavity through the first C3 channel (MPG)

Movie of the first C3 channel, along the whole trajectory, from the protein's cage point of view (MPG)

\section{AUTHOR INFORMATION}

\section{Corresponding Author}

*Tel.: +39055 4574267. E-mail: rosato@cerm.unifi.it (A.R.).

\section{ORCID $\odot$}

Paola Turano: 0000-0002-7683-8614

Antonio Rosato: 0000-0001-6172-0368

\section{Notes}

The authors declare no competing financial interest.

\section{ACKNOWLEDGMENTS}

This work was supported by Ministero dell'Istruzione, dell'Università e della Ricerca, PRIN 2012, Prot. 2012SK7ASN. S.C. acknowledges a postdoctoral grant by Fondazione Cassa di Risparmio di Firenze (no. 2013.0494) provided by FiorGen.

\section{ABBREVIATIONS}

$\mathrm{MD}$, molecular dynamics; $\mathrm{C} 3$, channel formed by three chains; C4, channel formed by four chains; TfR1, transferrin receptor 1; PDB, protein data bank; WT, wild type

\section{REFERENCES}

(1) Zhang, Y.; Orner, B. P. Self-Assembly in the Ferritin Nano-Cage Protein Superfamily. Int. J. Mol. Sci. 2011, 12 (12), 5406-5421.

(2) Bernacchioni, C.; Ghini, V.; Theil, E. C.; Turano, P. Modulating the Permeability of Ferritin Channels. RSC Adv. 2016, 6 (25), 2121921227.

(3) Chandramouli, B.; Bernacchioni, C.; Di Maio, D.; Turano, P.; Brancato, G. Electrostatic and Structural Bases of Fe2+ Translocation through Ferritin Channels. J. Biol. Chem. 2016, 291 (49), 2561725628.

(4) Theil, E. C.; Behera, R. K.; Tosha, T. Ferritins for Chemistry and for Life. Coord. Chem. Rev. 2013, 257 (2), 579-586. 
(5) Crichton, R. R.; Declercq, J.-P. X-Ray Structures of Ferritins and Related Proteins. Biochim. Biophys. Acta, Gen. Subj. 2010, 1800 (8), 706-718.

(6) Pozzi, C.; Di Pisa, F.; Bernacchioni, C.; Ciambellotti, S.; Turano, P.; Mangani, S. Iron Binding to Human Heavy-Chain Ferritin. Acta Crystallogr., Sect. D: Biol. Crystallogr. 2015, 71 (9), 1909-1920.

(7) Linder, M. C. Mobilization of Stored Iron in Mammals: A Review. Nutrients; Multidisciplinary Digital Publishing Institute, October 10, 2013; pp 4022-4050.

(8) Theil, E. C. Ferritin: The Protein Nanocage and Iron Biomineral in Health and in Disease. Inorg. Chem. 2013, 52 (21), 12223-12233.

(9) Finazzi, D.; Arosio, P. Biology of Ferritin in Mammals: An Update on Iron Storage, Oxidative Damage and Neurodegeneration. Arch. Toxicol. 2014, 88 (10), 1787-1802.

(10) Melman, G.; Bou-Abdallah, F.; Vane, E.; Maura, P.; Arosio, P.; Melman, A. Iron Release from Ferritin by Flavin Nucleotides. Biochim. Biophys. Acta, Gen. Subj. 2013, 1830 (10), 4669-4674.

(11) Hynes, M. J.; O Coinceanainn, M. M. Investigation of the Release of Iron from Ferritin by Naturally Occurring Antioxidants. J. Inorg. Biochem. 2002, 90 (1), 18-21.

(12) Kidane, T. Z. Release of Iron from Ferritin Requires Lysosomal Activity. AJP Cell Physiol. 2006, 291 (3), C445-C455.

(13) Lewis, H. A.; Buchanan, S. G.; Burley, S. K.; Conners, K.; Dickey, M.; Dorwart, M.; Fowler, R.; Gao, X.; Guggino, W. B.; Hendrickson, W. A.; Hunt, J. F.; Kearins, M. C.; Lorimer, D.; Maloney, P. C.; Post, K. W.; Rajashankar, K. R.; Rutter, M. E.; Sauder, J. M.; Shriver, S.; Thibodeau, P. H.; Thomas, P. J.; Zhang, M.; Zhao, X.; Emtage, S. Structure of Nucleotide-Binding Domain 1 of the Cystic Fibrosis Transmembrane Conductance Regulator. EMBO J. 2004, 23 (2), 282-293.

(14) Mancias, J. D.; Wang, X.; Gygi, S. P.; Harper, J. W.; Kimmelman, A. C. Quantitative Proteomics Identifies NCOA4 as the Cargo Receptor Mediating Ferritinophagy. Nature 2014, 509 (7498), 105-109.

(15) Asano, T.; Komatsu, M.; Yamaguchi-Iwai, Y.; Ishikawa, F.; Mizushima, N.; Iwai, K. Distinct Mechanisms of Ferritin Delivery to Lysosomes in Iron-Depleted and Iron-Replete Cells. Mol. Cell. Biol. 2011, 31 (10), 2040-2052.

(16) Li, L.; Fang, C. J.; Ryan, J. C.; Niemi, E. C.; Lebrón, J. A.; Björkman, P. J.; Arase, H.; Torti, F. M.; Torti, S. V.; Nakamura, M. C.; Seaman, W. E. Binding and Uptake of H-Ferritin Are Mediated by Human Transferrin Receptor-1. Proc. Natl. Acad. Sci. U. S. A. 2010, 107 (8), 3505-3510.

(17) Yamashiro, D. J.; Maxfield, F. R. Acidification of Morphologically Distinct Endosomes in Mutant and Wild-Type Chinese Hamster Ovary Cells. J. Cell Biol. 1987, 105 (6), 2723-2733.

(18) Maxfield, F. R.; Yamashiro, D. J. Endosome Acidification and the Pathways of Receptor-Mediated Endocytosis. Adv. Exp. Med. Biol. 1987, 225, 189-198.

(19) Tosha, T.; Behera, R. K.; Ng, H. L.; Bhattasali, O.; Alber, T.; Theil, E. C. Ferritin Protein Nanocage Ion Channels: Gating by NTerminal Extensions. J. Biol. Chem. 2012, 287 (16), 13016-13025.

(20) Bradley, J. M.; Le Brun, N. E.; Moore, G. R. Ferritins: Furnishing Proteins with Iron. J. Biol. Inorg. Chem. 2016, 21, 13-28.

(21) Haldar, S.; Bevers, L. E.; Tosha, T.; Theil, E. C. Moving Iron through Ferritin Protein Nanocages Depends on Residues throughout Each Four -Helix Bundle Subunit. J. Biol. Chem. 2011, 286 (29), $25620-25627$.

(22) Bertini, I.; Lalli, D.; Mangani, S.; Pozzi, C.; Rosa, C.; Theil, E. C.; Turano, P. Structural Insights into the Ferroxidase Site of Ferritins from Higher Eukaryotes. J. Am. Chem. Soc. 2012, 134 (14), 61696176.

(23) Roux, B. The Calculation of the Potential of Mean Force Using Computer Simulations. Comput. Phys. Commun. 1995, 91 (1-3), 275282.

(24) Rui, H.; Rivera, M.; Im, W. Protein Dynamics and Ion Traffic in Bacterioferritin. Biochemistry 2012, 51 (49), 9900-9910.

(25) Yao, H.; Rui, H.; Kumar, R.; Eshelman, K.; Lovell, S.; Battaile, K. P.; Im, W.; Rivera, M. Concerted Motions Networking Pores and
Distant Ferroxidase Centers Enable Bacterioferritin Function and Iron Traffic. Biochemistry 2015, 54 (8), 1611-1627.

(26) Yao, H.; Jepkorir, G.; Lovell, S.; Nama, P. V.; Weeratunga, S.; Battaile, K. P.; Rivera, M. Two Distinct Ferritin-like Molecules in Pseudomonas Aeruginosa: The Product of the bfrA Gene Is a Bacterial Ferritin (FtnA) and Not a Bacterioferritin (Bfr). Biochemistry 2011, 50 (23), 5236-5248. 